\title{
Enxerto subclávio-carotídeo como método de tratamento na obstrução da artéria carótida comum
}

\author{
Subclavian-carotid bypass graft for the occlusion of the common \\ carotid artery
Otacílio de Camargo Junior ${ }^{1}$, Luiz Roberto Felizzola ${ }^{2}$, Antonio Cláudio Guedes Chrispim², Claudio Roberto Cabrini Simões ${ }^{2}$, Márcia Fayad Marcondes ${ }^{3}$, Marivan Pedra Araújo ${ }^{3}$, Kelly Cristina Moraes ${ }^{3}$, Márcio Villar de Freitas ${ }^{3}$

\begin{abstract}
Resumo
A oclusão isolada da artéria carótida comum (ACC) é uma lesão relativamente incomum ( 0,5 a $5 \%)$. A maioria dos pacientes com obstrução da ACC tem lesão concomitante na artéria carótida interna (ACI) e na artéria carótida externa (ACE) ipsilaterais, sendo que, ocasionalmente, a circulação colateral da ACE pode preservar a perviedade da ACI via fluxo retrógrado. Relatamos o caso de um paciente sintomático com oclusão da ACC e perviedade das ACI e ACE tratado cirurgicamente com enxerto subclávio-carotídeo.
\end{abstract}

Palavras-chave: Enxerto subclávio-carotídeo, oclusão artéria carótida, doença cerebrovascular.

\section{Introdução}

A oclusão isolada da artéria carótida comum (ACC) é uma lesão relativamente incomum $(0,5$ a $5 \%$ dos pacientes com isquemia cerebral) ${ }^{1}$. A maioria dos pacientes com obstrução da ACC tem lesão concomitante na artéria carótida interna (ACI) e na artéria carótida externa (ACE) ipsilaterais. Ocasionalmente, a circulação colateral da ACE pode preservar a patência da $\mathrm{ACI}$ via perfusão retrógrada através do bulbo carotídeo ${ }^{2}$. Uma variedade de procedimentos é utilizada para a reconstrução do fluxo arterial nesse tipo de variante anatômica. As técnicas utilizadas incluem enxertos subclávio-carotídeo, axilar-carotídeo e carotídeo-carotídeo, todos com ou sem endarterectomia simultânea; endarterectomia carotídea com trombectomia da ACC; e enxerto aórtico-carotídeo ${ }^{2,3}$. O enxerto subclávio-carotídeo é o procedimento mais utilizado e o mais se-

\begin{abstract}
Isolated occlusion of the common carotid artery $(\mathrm{CCA})$ is a relatively uncommon lesion ( 0.5 to $5 \%$ ). Most patients with occlusion of the CCA have concomitant lesions of the ipsilateral internal carotid artery (ICA) and external carotid artery (ECA), and ECA may occasionally preserve ICA patency by means of retrograde flow. We report the case of a symptomatic patient with occlusion of the CCA and patency of the ICA and ECA treated with subclavian-carotid bypass graft.
\end{abstract}

Keywords: Subclavian-carotid bypass, carotid artery occlusion, cerebrovascular disease.

guro segundo a literatura ${ }^{1,2,4-6}$. Descreveremos um caso no qual foi utilizada tal técnica.

\section{Relato do caso}

Paciente masculino, 75 anos, hipertenso e tabagista, com história de acidente vascular cerebral isquêmico (AVCi) 3 meses antes, com sequela motora parcialmente revertida em membro superior direito. O AVCi foi comprovado por tomografia computadorizada (TC). O ultrassom Doppler de carótidas evidenciou oclusão da artéria carótida comum esquerda (ACCE); inversão do fluxo na artéria carótida externa esquerda (ACEE); artéria carótida interna esquerda (ACIE) com paredes espessadas e com fluxo proveniente da ACEE; artéria carótida comum direita (ACCD), artéria carótida interna direita (ACID) e artéria carótida externa direita (ACED) pérvias, porém com pare-

\footnotetext{
1. Professor adjunto. Chefe do Serviço de Angiologia e Cirurgia Vascular, Hospital e Maternidade Celso Pierro (HMCP), Campinas, SP.

2. Médico, Serviço de Angiologia e Cirurgia Vascular, HMCP, Campinas, SP.

3. Residente, Serviço de Angiologia e Cirurgia Vascular, HMCP, Campinas, SP.

Trabalho desenvolvido no Hospital e Maternidade Celso Pierro, Pontifícia Universidade Católica de Campinas, e apresentado no IX Panamerican Congress on Vascular and Endovascular Surgery.

Não foram declarados conflitos de interesse associados à publicação deste artigo.

Artigo submetido em 11.09.09, aceito em 29.10.09.

J Vasc Bras. 2010;9(1):78-81.

Copyright (C 2010 by Sociedade Brasileira de Angiologia e de Cirurgia Vascular
} 
des espessadas por placas calcificadas. Foi indicado o uso de antiagregante plaquetário, e foram mantidas as medicações anti-hipertensivas. Foi solicitada uma arteriografia que confirmou a oclusão da ACCE com circulação colateral cervical para a ACEE e enchimento da ACIE. As ACCD, ACID e ACED apresentavam-se com contorno irregular e com calibre preservados; foi identificada artéria vertebral direita dominante com estenose ostial crítica e artéria vertebral esquerda hipoplásica (Figura 1). Uma semana após a realização da arteriografia o paciente apresentou quadro de acidente isquêmico transitório novamente com deficit motor em membro superior direito, acompanhado de episódios de tontura. Foi mantido o uso de antiagregante plaquetário e de drogas anti-hipertensivas e solicitado novo ultrassom Doppler de carótidas, que evidenciou a patência da ACIE com fluxo proveniente da ACEE. O paciente foi submetido a endarterectomia da bifurcação carotídea (ACCE, ACIE e ACEE) com realização de enxerto subclávio-carotídeo à esquerda com prótese de politetrafluoretileno (PTFE) de $6 \mathrm{~mm}$ (Figuras 2 e 3). O procedimento cirúrgico foi realizado sob anestesia geral sem a necessidade de derivação temporária. O paciente evoluiu assintomático, recebendo alta hospitalar no terceiro dia pós-operatório. O ultrassom Doppler de carótidas e a arte-

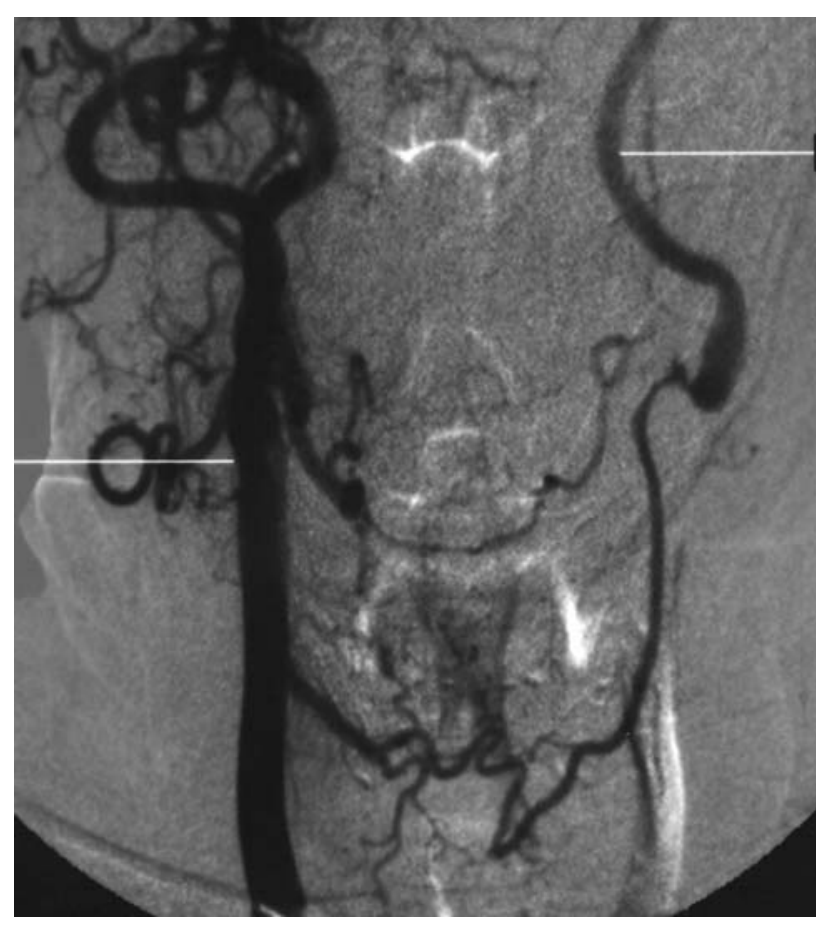

Figura 1 - Arteriografia: enchimento da artéria carótida esquerda através de circulação colateral da artéria carótida direita

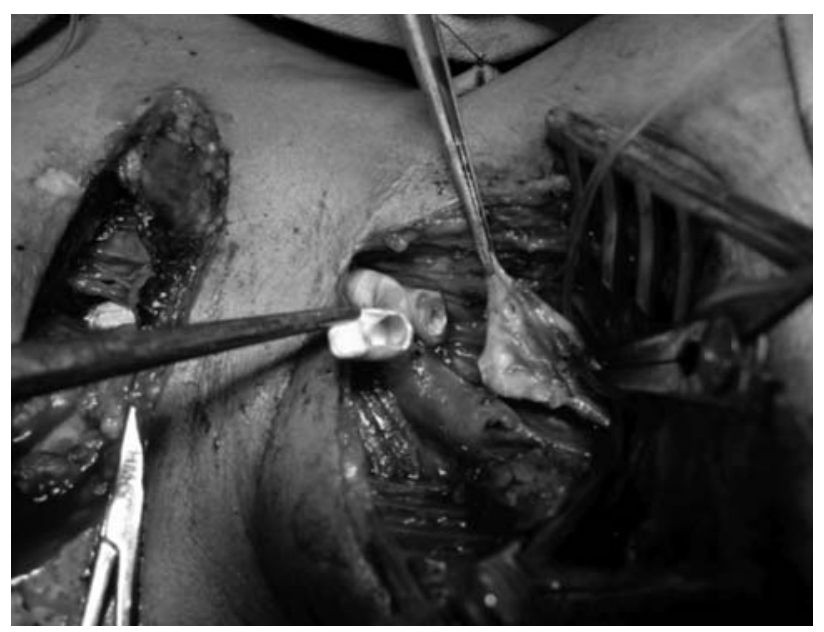

Figura 2 - Intraoperatório: artéria carótida comum esquerda seccionada, ocluída, e endarterectomia da bifurcação carotídea

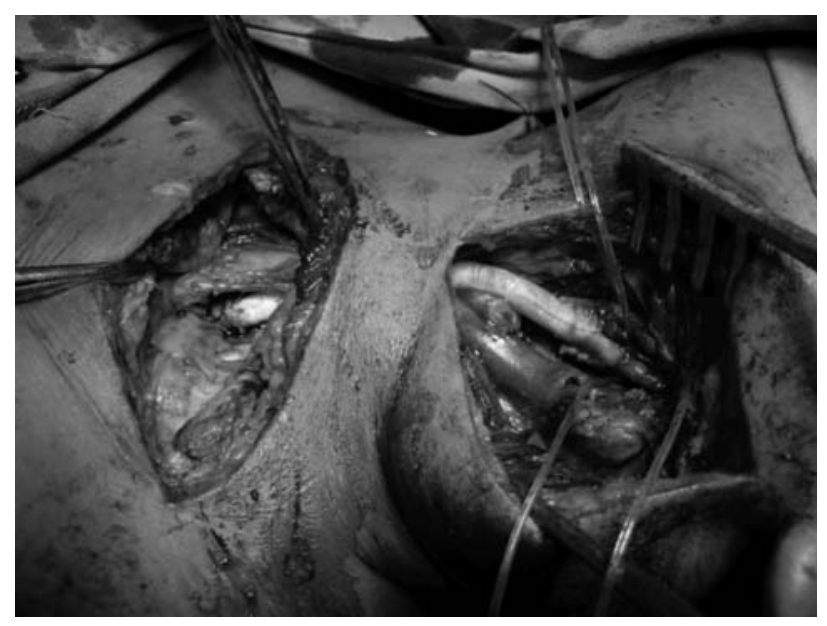

Figura 3 - Intraoperatório: enxerto subclávio carotídeo com prótese de politetrafluoretileno

riografia solicitadas 6 meses após a cirurgia evidenciaram a patência do enxerto subclávio-carotídeo à esquerda, mas com oclusão da ACEE e da artéria vertebral direita (Figura 4). O paciente permanece em acompanhamento ambulatorial há 2 anos, sem novos sintomas neurológicos.

\section{Discussão}

Embora a localização predominante da doença carotídea sintomática da artéria carótida seja na bifurcação carotídea, as lesões na ACC proximal causam sintomas similares $^{7}$. Os fatores de risco para doença oclusiva das artérias carótidas incluem tabagismo (76\%), hipertensão (71\%), diabetes melito (41\%) e hiperlipidemia (41\%). Em 82\% dos casos existem dois ou mais riscos incluídos. Doenças 


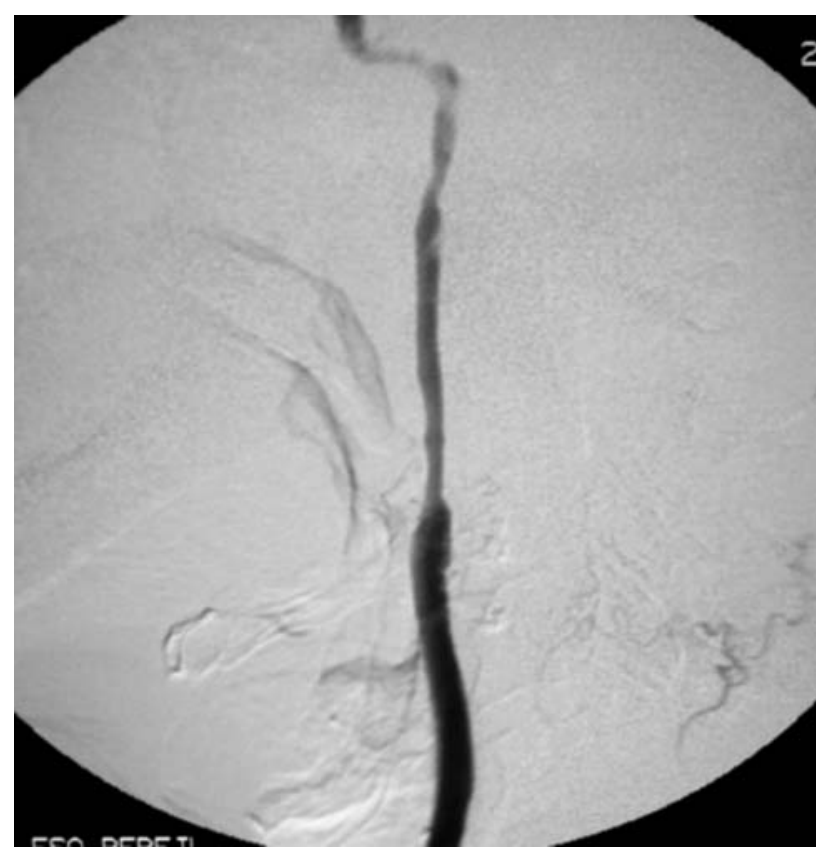

Figura 4 - Arteriografia: oclusão da artéria carótida externa e perviedade da carótida interna

cardíacas estão presentes em $59 \%$ dos pacientes ${ }^{8}$. Os mecanismos dos sintomas são multifatoriais e os mesmos da doença localizada na ACI e bifurcação carotídea ${ }^{9}$. Os pacientes acometidos por oclusão da ACC apresentam risco de ataques isquêmicos transitórios (AIT) hemisféricos, acidente vascular cerebral (AVC) ou sintomas vertebrobasilares $^{10}$.

Os sinais e sintomas predominantes incluem sintomas visuais monoculares e retroquiasmais ipsilaterais (88\%), perda de força motora (88\%), distúrbios sensoriais (59\%), amaurose fugaz (53\%) e síncope (24\%). Os AIT são geralmente múltiplos e precedem um AVC, ou ocorrem sem AVC subsequente em $82 \%$ dos $\operatorname{casos}^{8}$. Historicamente, o diagnóstico da oclusão da ACC era feito através de arteriografia cerebral ou exploração às cegas da bifurcação carotídea. Mais recentemente, o ultrassom Doppler tem sido utilizado para diagnóstico e permite localizar com precisão e apropriadamente a bifurcação carotídea ${ }^{1,10}$. As indicações cirúrgicas para obstrução da ACC incluem episódio de AIT, AVC, sintomas de insuficiência vertebrobasilar e sintomas não focais (síncope, vertigem) compatíveis com exame complementar ${ }^{4,5,11,12}$. A restauração do fluxo da artéria carótida através da artéria subclávia baseada num enxerto extra-anatômico providenciará uma reconstrução adequada para a obstrução da $\mathrm{ACC}^{7}$. Diversos estu- dos têm demonstrado que o enxerto subclávio-carotídeo é o procedimento mais utilizado na oclusão da $\mathrm{ACC}$ e uma excelente opção cirúrgica, pois apresenta bons resultados a longo prazo e redução significativa dos sintomas no pósoperatório ${ }^{1,3,10}$. Em relação ao tipo de enxerto utilizado, Ziomek et al. ${ }^{6}$ obtiveram melhores resultados com o uso de prótese $(91 \%$ dos enxertos patentes nos casos em que foi utilizada prótese e $57 \%$ nos casos com veia). Vitti et al. ${ }^{5}$ realizaram estudo retrospectivo com 124 pacientes nos quais o enxerto com prótese foi utilizado em todos os casos (35\% PTFE e 65\% dácron) com taxa de patência de $95 \%$ em 5 anos e taxa de sobrevivência sem sintomas de $90 \%$ em 5 anos. Já Defraigne et al. ${ }^{9}$ realizaram enxerto subclávio-carotídeo em 29 pacientes. Em 15 casos, foi utilizada a veia safena, e 14 foram feitos com prótese, não havendo diferença na patência entre os materiais utilizados $(89 \%$ em 40 meses para ambos). Salam et al. ${ }^{4}$ utilizaram veia safena em $65 \%$ dos enxertos realizados e obtiveram taxa de patência de $90 \%$ em 3 anos. Enfim, reconhecer a patência distal das artérias acima da oclusão da ACC depende de um alto índice de suspeição, investigação cuidadosa do bulbo carotídeo com duplex scan e/ou arteriografia para investigação do reenchimento através das artérias colaterais $^{2}$. Conclui-se, portanto, que pacientes com oclusão da ACC e perviedade acima da oclusão podem ser tratados cirurgicamente através da confecção de enxerto subclávio-carotídeo. Este procedimento mostrou-se, pelas publicações estudadas, seguro, efetivo, com baixa morbidade e mortalidade e com bons resultados no seguimento, independentemente do tipo de material utilizado para o enxerto, sendo o mais comumente realizado na atualida$\mathrm{de}^{1,3-5,9,10}$.

\section{Referências}

1. Adovasio R, Visitin E. Il by-pass succlavio-carotideo quale metódica di trattamento dell'ostruzione della carotide comune. Ann Ital Chir. 1999;70:247-50.

2. Belkin M, Mackey WC, Pessin M, et al. Common carotid occlusion with patent internal and external carotid arteries: diagnosis and surgical management. J Vasc Surg. 1993;17:1019-28.

3. Dellaretti Filho MA, de Sousa AA, Carvalho GT; de Castro MF. Occlusion of the common carotid artery treated with a subclavian-internal carotid artery bypass. Arq Neuropsiquiatr. 2003;61:453-5.

4. Salam TA, Smith RB 3rd, Lumsden AB. Extrathoracic bypass procedures for proximal common carotid artery lesions. Am J Surg. 1993;166:163-7. 
5. Vitti MJ, Thompson BW, Read RC, Gagne PJ, Barone GW, Barnes RW, Eidt JF. Carotid-subclavian bypass: a twentytwo-year experience. J Vasc Surg. 1994;20:411-8.

6. Ziomek S, Quiñones-Baldrich WJ, Busuttil RW, et al. The superiority of synthetic arterial grafts over autologous veins in carotid-subclavian bypass. J Vasc Surg. 1986;3:140-5.

7. Fry WR, Martín JD, Clagett GP, et al. Extrathoracic carotid reconstruction: the subclavian-carotid artery bypass. J Vasc Surg. 1992;15:83-9.

8. Levine SR, Welch KMA. Common carotid artery occlusion. Neurology. 1989;39:178-86.

9. Defraigne JO, Remy D, Creemers E, Limet R. Carotid-subclavian bypass with or without carotid endarterectomy. Acta Chir Belg. 1990;90:248-54.

10. Sullivan TM. Subclaviacarotid bypass to an "isolated" carotid bifurcation: a retrospective analysis. Ann Vasc Surg. 1996;10:283-9.

11. Martín SR 3rd, Edwards WH, Mulherin JL. Surgical treatment of common carotid artery occlusion. Am J Surg. 1993;165:302-6.

12. Perler BA, Williams GM. Carotid-subclavian bypass: a decade of experience. J Vasc Surg. 1990;12:716-23.
Correspondência:

Otacílio de Camargo Junior

Rua Cândido Gomide, 468, Jd Guanabara

CEP 13073-200 - Campinas, SP

Tel.: (19) 3243.8090

E-mail: otacamjr@terra.com.br

Contribuições dos autores

Concepção e desenho do estudo: OC Junior

Análise e interpretação dos dados: OC Junior, ACGC, CRCS

Coleta de dados: KCM, MVF, MPA, MFM

Redação do artigo: OC Junior

Revisão crítica do texto: OC Junior, MFM, LRF, KCM

Aprovação final do artigo*: OC Junior, ACGC, CRCS, MFM, LRF, KCM, MVF, MPA

Análise estatística: OCJ

Responsabilidade geral pelo estudo: OC Junior

Informações sobre financiamento: OC Junior

* Todos os autores leram e aprovaram a versão final submetida ao J Vasc Bras. 